\title{
Delivery Considerations of Highly Viscous Polymeric Fluids Mimicking Concentrated Biopharmaceuticals: Assessment of Injectability via Measurement of Total Work Done " $W_{\mathrm{T}}$ "
}

\author{
Qiangnan Zhang, ${ }^{1}$ Mona A. Fassihi, ${ }^{2}$ and Reza Fassihi ${ }^{1,3}$
}

Received 25 August 2017; accepted 23 January 2018; published online 20 February 2018

\begin{abstract}
An account is given of the recent development of the highly viscous complex biopharmaceuticals in relation to syringeability and injectability. The specific objective of this study is to establish a convenient method to examine problem of the injectability for the needle-syringe-formulation system when complex formulations with diverse viscosities are used. This work presents the inter-relationship between needle size, syringe volume, viscosity, and injectability of polymeric solutions having typical viscosities encountered in concentrated biologics, by applying a constant probe crosshead speed on the plunger-syringe needle assembly and continuously recording the force-distance profiles. A computerized texture analyzer was used to accurately capture, display, and store force, displacement, and time data. The force-distance curve and area under the curve are determined, and total work done for complete extrusion of the syringe content was calculated automatically by applying an established Matlab program. Various concentrations (i.e., $0.5-4 \% w / v$ of polymeric fluids/ dispersions) of polyethylene oxide (PEO) and hydroxypropyl methylcellulose (HPMC) with viscosity ranges of 5-100 $\mathrm{cP}$ mimicking concentrated monoclonal antibody solutions and complex biopharmaceutical formulations are investigated. Results indicate that calculated values of total work done to completely extrude the syringe content are the most appropriate parameter that describes viscosity-injection force of dispersed formulations. Additionally, the rheological properties of HPMC and PEO fluids in the context of syringeability and injectability are discussed.
\end{abstract}

KEY WORDS: syringeability; injectability; HPMC; PEO; biologics.

\section{INTRODUCTION}

Syringeability (i.e., ease of withdrawal from vial to syringe) and injectability (i.e., formulation performance during injection including pre-filled syringes) are two very important constraints in handling highly concentrated and complex formulations of recombinant biologics, proteins, and biopharmaceutical drugs often not experienced with conventional small molecule simple parenteral (1). Biologics having high molecular weights (i.e., insulin $5.8 \mathrm{kDa}$; Humira consisting of 1330 amino acids, and MW $148 \mathrm{kDa}$ ) and high concentrations per milliliter $(\mathrm{mg} / \mathrm{mL})$ and their means of delivery as opposed to small molecular weight drugs (i.e., epinephrine $183 \mathrm{Da}$ ) are associated with many challenges including their rheology. With advances in formulation

\footnotetext{
${ }^{1}$ School of Pharmacy, Temple University, 3307 North Broad St., Philadelphia, Pennsylvania 19140, USA.

${ }^{2}$ Novartis Pharmaceuticals Corporation, One Health Plaza, East Hanover, New Jersey 07936-1080, USA.

${ }^{3}$ To whom correspondence should be addressed. (e-mail: reza.fassihi@temple.edu)
}

design, the complexity of the formulations that are designed to enhance the solubility of insoluble drugs and provide greater stability for concentrated biologicals which tend to undergo aggregation, deamination, denaturation, hydrolysis, or oxidation has also changed dramatically (2-4). Multiple excipients, agents, and environmental factors have been investigated that can ensure both physical and chemical stability of biopharmaceuticals including the use of small sugars such as trehalose $(5,6)$, dextran (7), surfactants like pluronic, polysorbate (8), and influence of $\mathrm{pH}$, temperature, and ionic strength. Although peptides and proteins have high specificity and potency, in many cases, concerns related to the high dose of proteins with varying viscosities as often concentrated formulations with several hundred milligrams per milliliter are required especially to satisfy the limited volume allowed for subcutaneous injection as often preferred by FDA and patients. As early as 1979, Ritschel and Suzuki (9) and Dexter and Schott (10) proposed methods and procedures to determine force required to smoothly inject suspensions or oily parenteral into tissues for a given syringeneedle system. The high viscosity of the formulation affects both syringeability and formulation performance during 
injection, necessitating more force with an acceptable needle gauge and time needed. Presently, intravenous, intramuscular, and more convenient subcutaneous route is the most frequently used methods of delivering biologicals. Drug dosages and delivery systems for biopharmaceuticals include emulsions, suspensions, liposomes, micro-emulsions, degradable microspheres, antibody drug conjugates (ADCs), recombinant biologicals, implants, extended release, and highly viscous and/or thixotropic gel systems (1). Approximate size spectrum showing dimensions of typical molecules, carrier types and drug delivery systems, and some of the commercialized biopharmaceutical products is presented in Fig. 1.

It is imperative to consider formulation performance in the context of needle-syringe-formulation-trio system when designing biopharmaceutical formulations. Development of the biologic formulations is significantly different to that of small molecule formulation (i.e., non-Newtonian in the former and Newtonian in the latter case) and it requires addition of multiple components and an in-depth understanding of the formulation design to assure maximum stability and manufacturability, with full considerations of viscosity and dynamics of stress-strain shearing effects in the context of rheological behavior. For example, it has been reported that, changes in viscosity of highly concentrated formulations of humanized monoclonal antibodies (mAbs) were linked to multivalent and concentration-dependent reversible self-association of a mAbs and electrostatic interactions in the presence of chaotropic anions impacting solution viscosity in an exponential mode (see Fig. 2) (3). Authors used a cone and plate rheometer, and viscosity measurements were performed by two cycles of shear-rate sweeps under defined conditions (3).

In this work, the term "viscosity" or "rheology" refers to the flow property or thickness of a liquid formulation. Typically, viscosities of liquids range from one to several thousand centipoise (cPs). For example, viscosity of water is $\sim 1-5 \mathrm{cP}$, blood is $10 \mathrm{cP}$, corn syrup $50-100 \mathrm{cP}$, maple syrup 150-200 cP, castor oil $250-500 \mathrm{cP}$, and many concentrated biologicals have viscosities of 10-200 cPs (4,11-13).

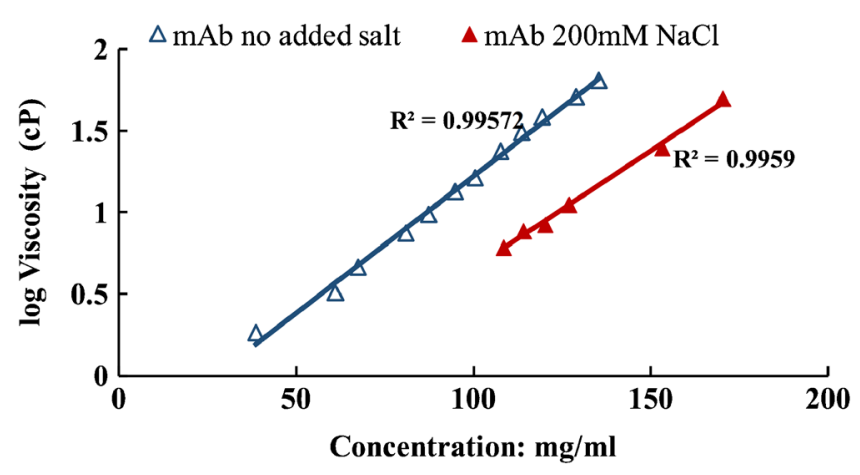

Fig. 2. Changes in solution viscosity of mAbs in $30 \mathrm{mM}$ histidine buffer at $\mathrm{pH} 6.0$, with and without added salt in the formulations

Viscosity is always influenced by formulation composition. Einstein (14) recognized that in the dispersed systems, single particles enhanced the viscosity of a liquid as a simple function of their phase volume, according to the following relationship (Eq. 1):

$\eta=\eta_{\text {medium }}(1+2.5 \phi) \ldots \ldots$

where $\eta$ is the viscosity of the suspension, $\eta_{\text {medium }}$ is the viscosity of the medium, and $\Phi$ is the volume fraction of solids in the suspension. This finding has great value for describing systems containing very low particle concentrations. However, in many biologics and biopharmaceutical formulations, particle loadings are high with greater proximity to each other and thus higher viscosity. Krieger and Doughetry (15) developed a semi-empirical model to account for this “crowding" effect (Eq. 2):

$\frac{\eta}{\eta_{\text {medium }}}=\left(1-\frac{\eta}{\eta_{\text {medium }}}\right)^{-[\eta] \phi_{\mathrm{m}}} \ldots \ldots$

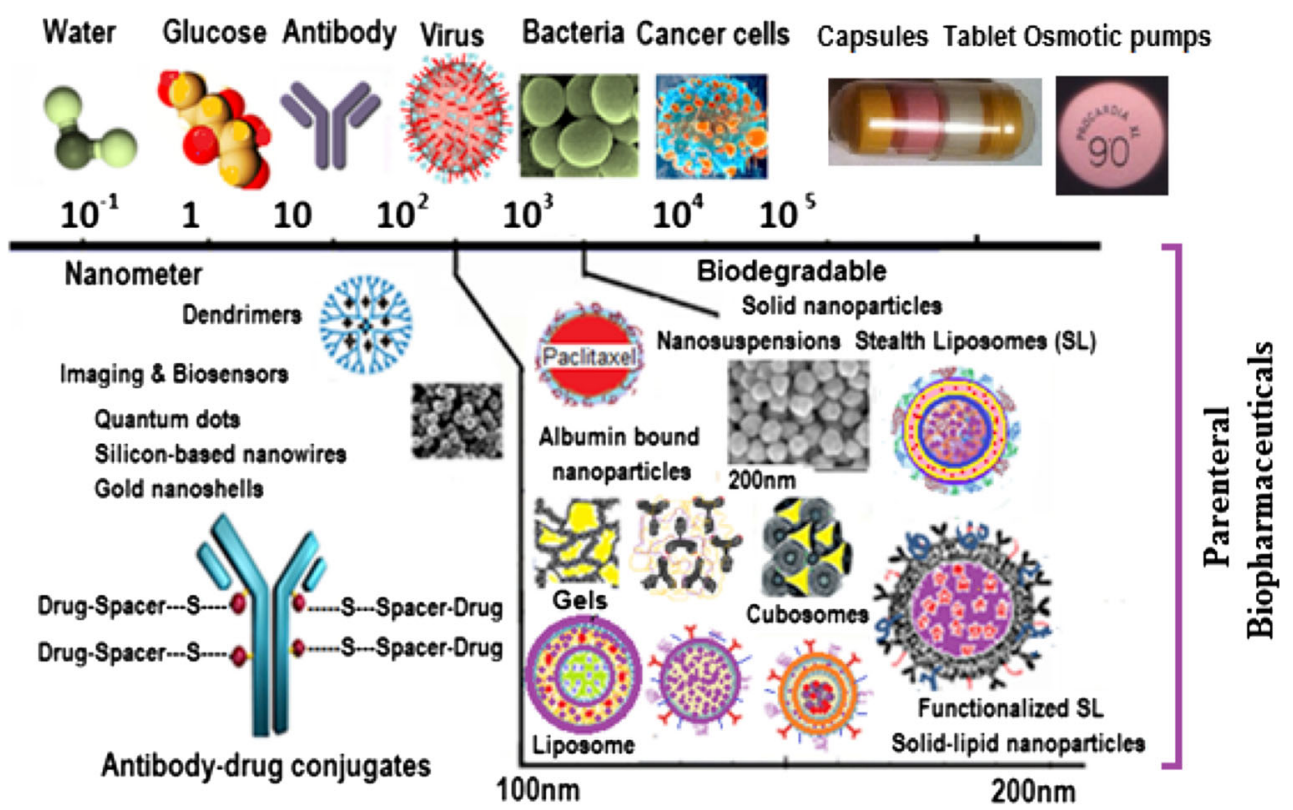

Fig. 1. Approximate size spectrum showing dimensions of typical molecules, carrier types and drug delivery systems, and some of the commercialized parenteral biopharmaceutical products 
Where $\Phi_{\mathrm{m}}$ is the maximum volume fraction of solids in the system, $[\eta]$ is the intrinsic viscosity with value of 2.5 for spheres. Addition of $\Phi_{\mathrm{m}}$ parameter shows that there is a maximum volume of particles that can be added before the system becomes too viscous.

Some examples of complex formulations with high viscosity and drug concentration currently on the market include biodegradable polymer-liquid carrier Eligard (leuprolide acetate) (16), Hyalgan (intra-articular injections based on sodium hyaluronate); Gel-One (cross-linked hyaluronate); Supartz FX (highly purified hyaluronic acid) (17), IM injection of oily system Faslodex (fulvestrant used in metastatic breast cancer), Lupron Depot extended release, highly concentrated recombinant interleukin-1 receptor antagonist (Kineret, anakinra $150 \mathrm{mg} / \mathrm{mL}$ ), and a fully biodegradable D,L-lactic-glycolic acids copolymer-based cylindrical implant Zoladex (goserelin acetate used in prostatic carcinoma, a pre-loaded 1.5 -mm-diameter cylinder in a special single-use syringe with a 14-gauge siliconized needle (2.11$\mathrm{mm}$ outer diameter) for subcutaneous delivery of implant, see Fig. 3).

In many cases, these products have range of viscosities and compositions. It is likely that two or more formulations might have similar viscosity; however, depending on the nature of the formulations and its rheological behavior, syringeability among different formulations may vary significantly. Hyalgan (sodium hyaluronate) formulation is a thixotropic gel system hence viscosity of the formulation is complex and decreases during the syringing process. On the other hand, Eligard (leuprolide acetate suspension) makes use of the shear thinning property of poly (lactic-co-glycolic acid) (PLGA $(18,19)$ ) solution to enhance the performance during injection period (ease of injectability).

The rheology or likely thixotropic characteristics of such biologics and complex formulations in terms of stress-strain observed among the products vary especially when subjected to application of plunger force (shear stress) and consequently shear rate while the content of the syringe is extruded (shear rate and flow velocity). Typical changes in flow and thixotropic characteristics of dispersed systems in general when subjected to stress are illustrated in Fig. 4.

Both FDA and ICH Q6A "Guidance for Industry" on container-closure and pre-filled syringes for human drugs and

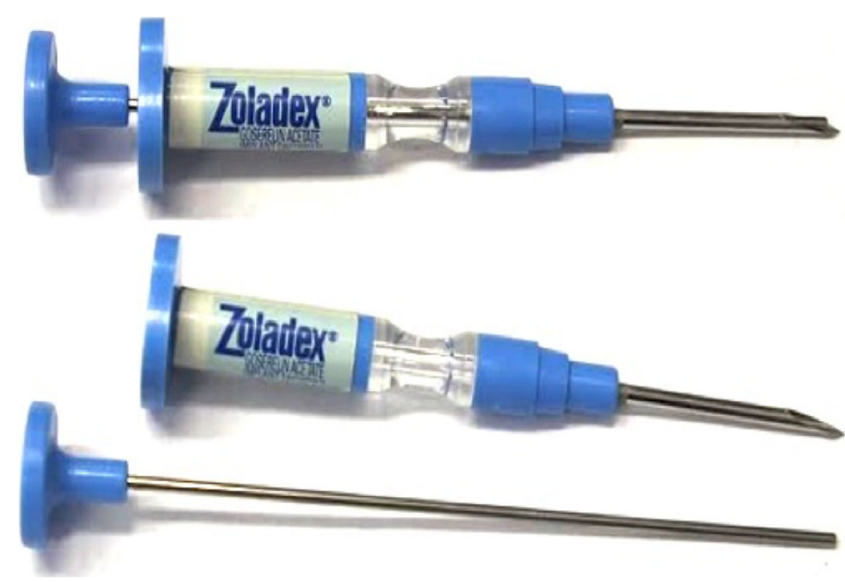

Fig. 3. Prefilled syringe containing a semisolid cylindrical implant for subcutaneous delivery
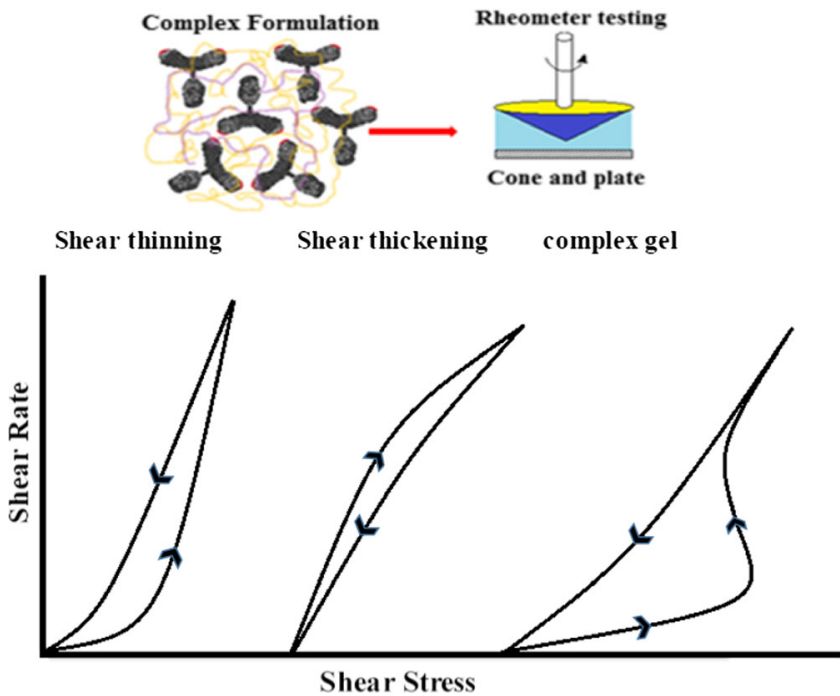

Fig. 4. Typical changes in shear rate with increasing shear stress observed for various structured liquids measured with a laboratory rheometer (cone and plate) showing shear thinning, shear thickening, and complex gel system behavior

biologics require syringe's performance, test procedures, and acceptance criteria related to the functionality of the delivery system $(20,21)$.

In order to determine the relationship between viscosity of the formulation, syringe type, and needle size, one could calculate the plunger force applied to the formulation to fully extrude the material through a needle comparable to injectability performance (22).

Theoretically, various viscous solutions emulating real drug-product rheology in terms of syringeability and/or injectability can be measured directly using the HagenPoiseuille equation, which is used to assess required force across a pipe or syringe to cause flow (23) (see Eq. 3):

$F=\frac{128 Q L \mu A}{\pi D^{4}} \ldots \ldots$

where $F$ is the force (i.e., syringe plunger force), $Q$ is the volumetric flow rate, $\mu$ is the viscosity of the fluid (i.e., formulation), $L$ is the length (displacement or distance traveled), $D$ is the diameter of the pipe/needle bore diameter, and $A$ is the area (i.e., syringe plunger area).

The purpose of this study is to evaluate the interrelationship between needle size, syringe volume, concentration, and injectability of the formulations by applying a constant machine probe crosshead speed on the plungersyringe needle assembly and continuously recording the force-displacement pattern. To this end, a computerized texture analyzer was used to accurately capture, display, and store force, displacement, and time data. The force-distance curve and area under the curve are determined, and total work (force-displacement) done for complete extrusion of the syringe content was calculated. Various fluids with different concentrations (i.e., $0.5-4 \% w / v$ ) of polyethylene oxide (PEO) and hydroxypropyl methylcellulose (HPMC) having viscosity range of 5-100 $\mathrm{cP}(24,25)$, mimicking viscosity 
encountered with many concentrated biopharmaceutical formulations, are investigated.

\section{MATERIALS AND METHODS}

\section{Materials and Equipment}

Texture analyzer (Texture Technologies Corp., Scarsdale, NY) was used to evaluate and quantify the total force of injectability performed on a needle-syringeformulation assembly. HPMC (Methocel, E5 PREM LV, The Dow Chemical Corp.) and PEO (WSRN-80, 200,000mw, Union Carbide Corp.) were used as a model dispersed system to calculate and mimic the work of injection. The dispersed fluids were prepared at a concentration of $0.5,1,2$, and $4 \% w / v$. The $3-\mathrm{mL}$ (AHS, AH03L) and 6-mL (Covidien, 8881516937) disposable syringes with the barrel diameter of 9 and $13 \mathrm{~mm}$ respectively are used. These were fitted with needle 1 (18G, 0.84-mm bore diameter and $43 \mathrm{~mm}$ in length), needle $2(21 \mathrm{G}, 0.51-\mathrm{mm}$ bore diameter and $43 \mathrm{~mm}$ in length), or without a needle and were used to measure either force of injection or total work of injection to extrude the syringe content completely.

\section{Texture Analysis to Collect Force-Distance Data}

The measure of injectability was investigated using a texture analyzer (XT2i) which was calibrated for force and distance measurement. The syringe is positioned in a holder shown in Fig. 5. A corresponding probe to match the surface area of the plunger plate was used to extrude the fluid volume of the syringe. During the test, the probe of the texture analyzer travels downward at a speed of $1 \mathrm{~mm} / \mathrm{s}$, forcing the content of the syringe out and producing the force-distance plot. The experiments were repeated three times.
The Study of Maximum Force, Dynamic Glide Force, and Total Work of Injection

The measurement of the injectability was defined as the ease of injection, represented by the injection force. The typical force distance-plot is shown in Fig. 6, which shows the profile of $4 \%(w / v)$ HPMC in 3-and 6-mL syringes without a needle attached. In order to evaluate the injectability, the maximum force and the dynamic glide force were identified and calculated from the force-distance plot using Matlab code. The maximum force $\left(F_{\max }\right)$ is the highest force before all the content is extruded. The dynamic glide force (DGF) (26) is the steady force which is required to sustain the movement of the fluid out of the syringe. With the help of Matlab coding, the DGF is determined based on the average value of the longest force range where any two points of force differences are within experimental noise threshold (27). Due to the large amount of data collection per run (more than 10,000 points recorded by texture analyzer besides two typical points of $F_{\max }$ and DGF) and variations in needle gauges and syringe body materials, we have determined that the total work of injection $\left(W_{\mathrm{T}}\right)$ more accurately characterizes extrusion of syringe content rather than simple measurement of force used to extrude the content.

\section{The Study of the Work of Injection}

To calculate the work of injection, the beginning and the end of injection based on force-distance were determined. The first point of injection is generated when the probe touches the plunger of the syringe. The last point is produced when all the content is fully extruded. Due to the high sensitivity of texture analyzer, the measured force-distance is not a smooth curve as slight resistance to flow or frictional forces can easily cause departure from linearity. Besides, due to the high rate of data collection during the testing with texture analyzer (one point per $0.005 \mathrm{~mm}$ ), simple curve fitting can lead to significant fitting error. To address these concerns, we implemented a Matlab code which can determine a short partial curve which contains the last point (28).

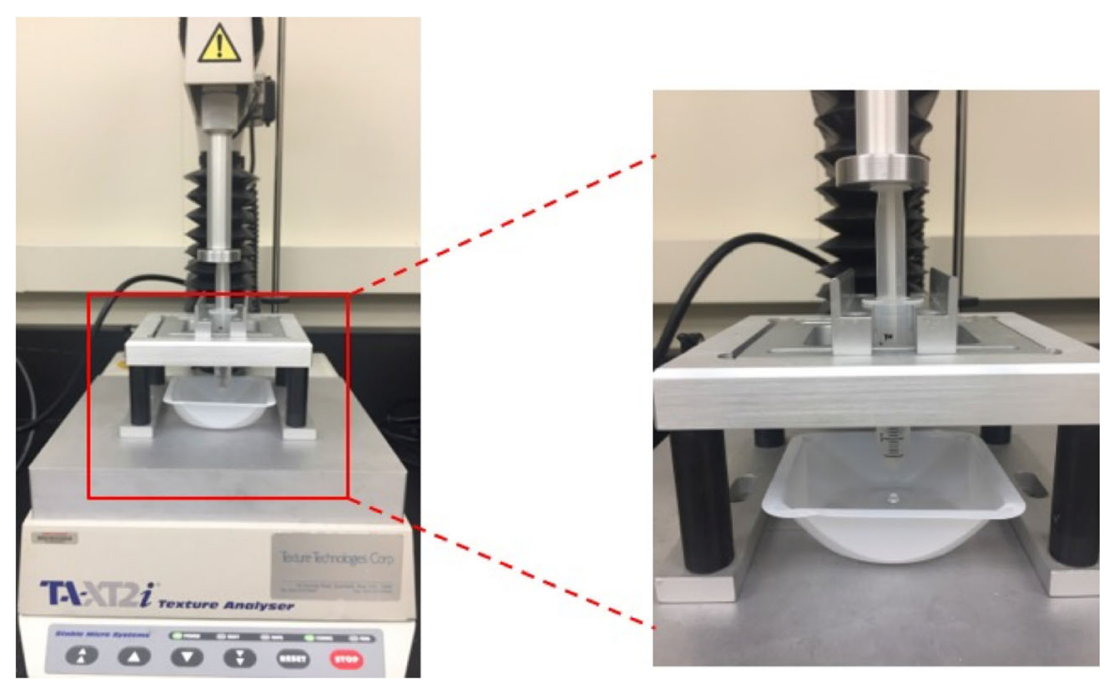

Fig. 5. Analog system of injection carried out by a software-controlled texture analyzer in compression mode 


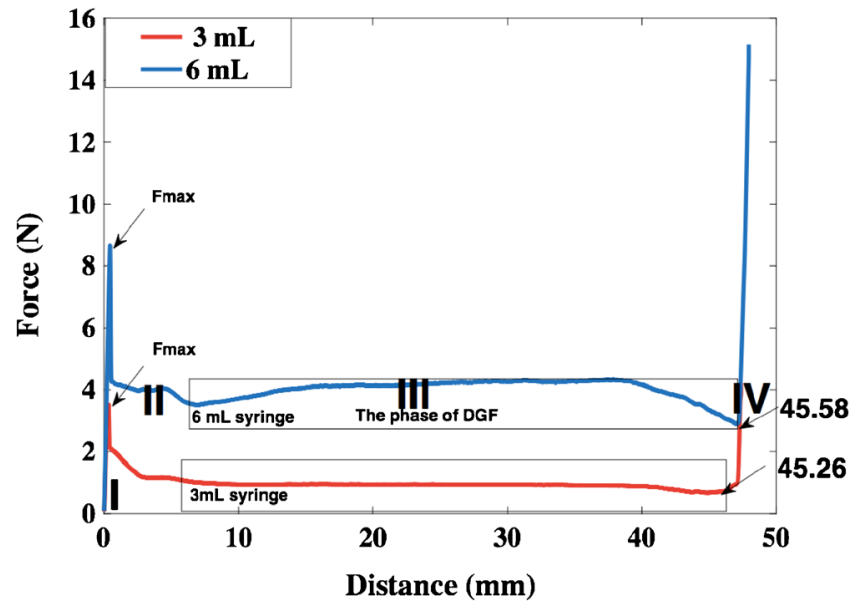

Fig. 6. Force-distance profile for a $4 \%(W / V)$ HPMC in 3- and 6-mL syringes with no needle attached

Then, we conduct curve fitting in this short partial curve. Therefore, the last point can be determined through the derivation of fitted curve, and the error is minimized. To achieve this, a Matlab code is developed to import collected data for further processing. The numbers pointed to in Fig. 6 (i.e., 45.26 and 45.48) are representative of the exact distance traveled where all the content has been extruded. The boxed area in both plots in Fig. 6 describes the entire distance that was used to calculate DGF and the calculation of the total work done to extrude the syringe content was based on the boxed distance.

\section{RESULTS}

Figure 5 shows a typical experimental setting with a flatended probe with large enough surface area to uniformly force the syringe plunger downward in the syringe barrel. Figure 6 shows the force-distance profiles obtained using 4\% $w / v$ solution of HPMC at room temperature when extruded through a 3- and 6-mL syringe with no needle attached. Profiles show that complete extrusion of the syringe content can be described by four distinct phases. Phase-I or $F_{\max }$ is the force applied to the plunger and detected by the system referred to as the "break loose force" which appears to be the maximum force $\left(F_{\max }\right)$ leading to phase-II where a sharp drop toward a steady state follows. This initial two phases constitute the "break-loose force" leading to phase-III, a pseudo-steady phase referred to as "dynamic-glide-force" (DGF) which is required to sustain the movement of the fluid while content is extruded. The last, phase-IV, is calculated using the Matlab coding approach representing the end of the injection (extrusion) which occurs at 45.58- and 45.26-mm distance for 6-and 3-mL syringes respectively. The final sharp rise in force signifies that the plunger has reached the end of the syringe barrel after all the content is extruded. Similar trends were also observed for other viscosity levels using both 3-and 6-mL syringes including use of the two needle gauges.

Figures 7 and 8 show the dynamic glide force "DGF" and the injection force " $F_{\max }$ " for HPMC and PEO solutions respectively as determined by the texture analyzer. Measurement of injection forces indicates that the $F_{\max }$ and DGF increase as a function of the viscosity of the polymeric solutions. In addition, with the decrease in the needle size and bore diameter, there is an increase in $F_{\max }$ and DGF. Overall, it appears that there is an increasing general trend among the polymeric solutions with increasing concentrations. Both DGF and the $F_{\max }$ under all conditions including use of needles with both 3- and 6-mL syringes show the same trend. It is also important to note that at certain concentrations, $F_{\max }$ values are not sensitive enough to clearly demonstrate the differences. For example, when $2 \%(w / v)$ HPMC solution with 3-mL syringe was used (see Fig. 8a), the comparison of the $F_{\max }$ values between needle 1 , needle 2, and without a needle is fairly small. The actual differences between them being only $0.1 \mathrm{~N}$. As can be seen in this case, using the index of injection force " $F_{\max }$ " which is also used by others (26) does not accurately differentiate between various settings, and this parameter seems not to be descriptive enough as a measure of injectability.

As a result of this observation, we have added a more significant and descriptive parameter based on the AUC of the force-distance (F-D) profiles (see Fig. 6) representing total work done $\left(W_{\mathrm{T}}\right)$ to completely extrude the syringe content. The total work involved, $W_{\mathrm{T}}$, was obtained by monitoring probe force " $F$ " and distance " $D$ " through which it acted. Using Eq. 4:

$W_{T}=\int_{D_{F=0}}^{D_{\max }} F \cdot d D \ldots \ldots$

By using this equation, we have calculated the AUC "area under the entire force-distance plot" representing the total work done " $W_{\mathrm{T}}$ " to extrude the syringe content. $D$ indicates the entire moving distance of the probe from the beginning to the moment that the syringe content is fully extruded. This distance and force was automatically captured and stored by the written Matlab program (described previously, see "Materials and Methods") using different syringes and solution concentrations with and without needles of different bore diameters but constant length. The use of the concept of total work $\left(W_{\mathrm{T}}\right)$ of injection was performed to compare behavior of different syringe-needle-formulation systems. The results for complete extrusion of both HPMC and PEO solutions are presented in Tables I and II. Figure 9 shows the comparative plots of the total work done for various settings. The total work done in the case of $6-\mathrm{mL}$ syringe is approximately three times greater than that of 3 -mL syringe using the same needle gauge. The range of injection work for 3-mL syringe is from about 20 to $100 \mathrm{~J}$, while for the 6-mL syringe, this range is from 100 to $400 \mathrm{~J}$ as shown in Fig. 9. These results clearly indicate that syringe volume or barrel size in addition to the rheology of the formulation has a disproportionate increase in total work done to extrude the syringe content. This observation is consistent with that of others who have shown existence of exponential relationship between viscosity increases of model antibody formulations as concentration of antibody increased $(3,4)$.

This trend also shows that the higher the concentration of the polymeric solution, a more disproportionate increase in the total work of injection especially as the needle bore diameter decreases. It appears that the total work of injection with different needle sizes and syringe 
a

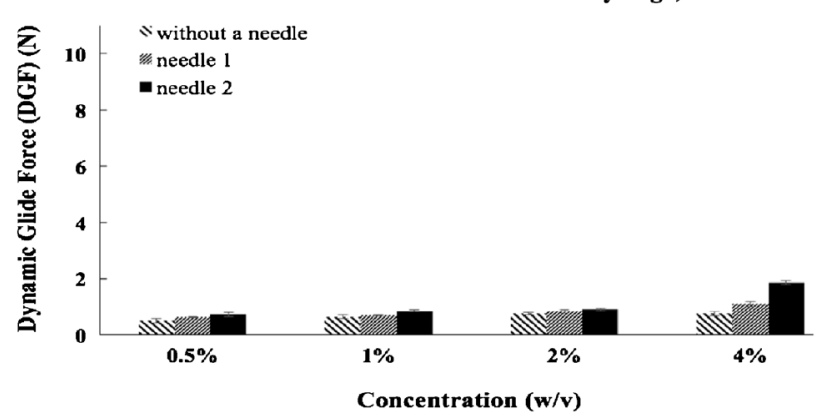

d

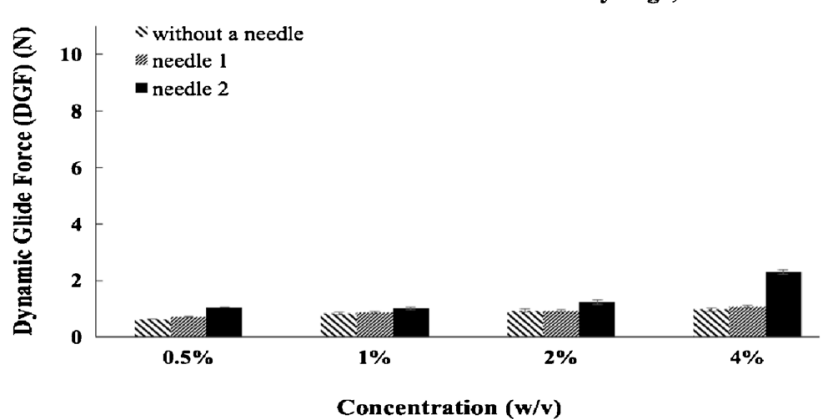

b

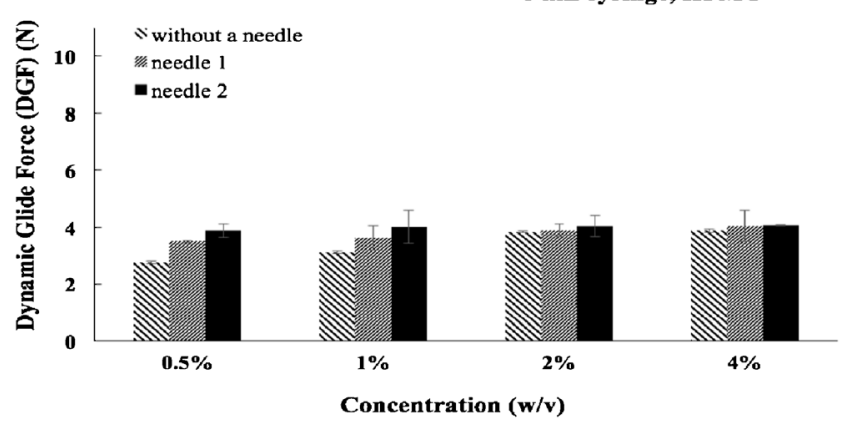

C

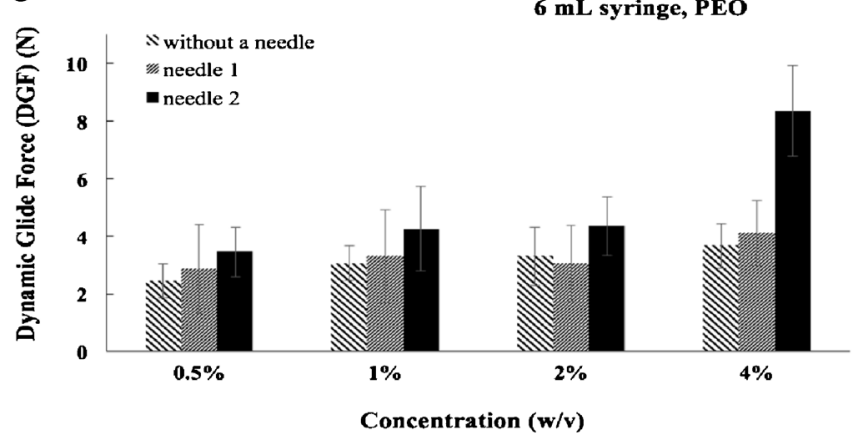

Fig. 7. Dynamic glide force (DGF) values for different solution concentrations of HPMC and PEO using 3- and 6-mL syringes with and without needles $(N=3)$. The results are expressed as the mean of three experiments \pm standard deviation

volumes is significantly impacted as concentration of the polymeric fluids increases. Profiles in Fig. 9 clearly show that the total work done when needle 2 is attached is always greater than that with needle 1 and this effect is more pronounced with PEO solutions at higher

a

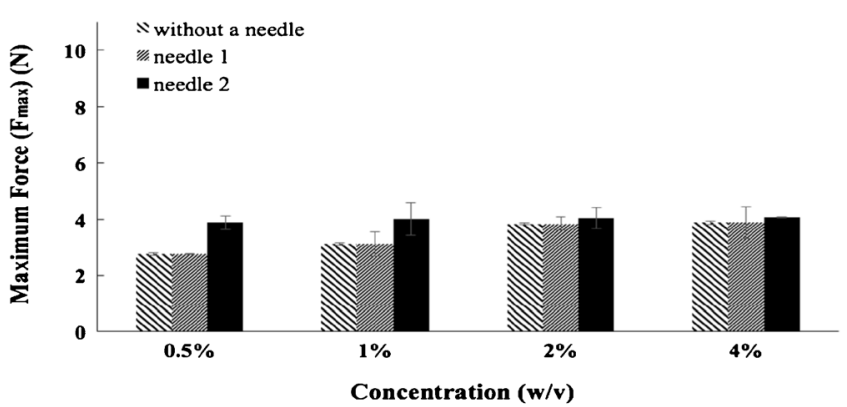

C

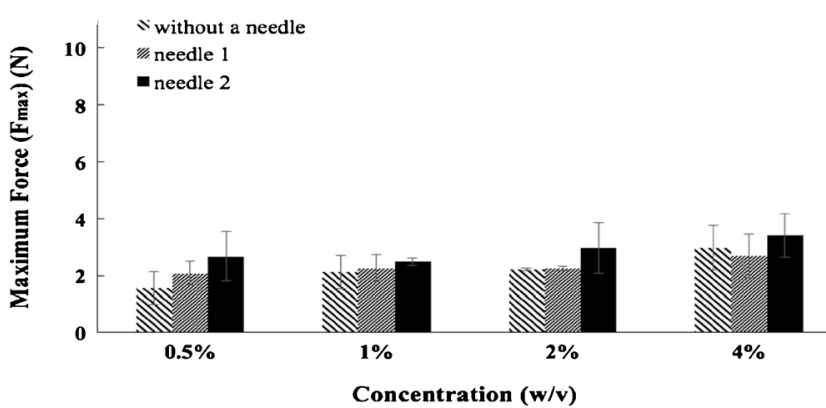

concentrations. These data suggest that the work of injection increases as the syringe volume and concentration of the solution increases and this effect disproportionately increases as the needle bore diameter becomes smaller. b

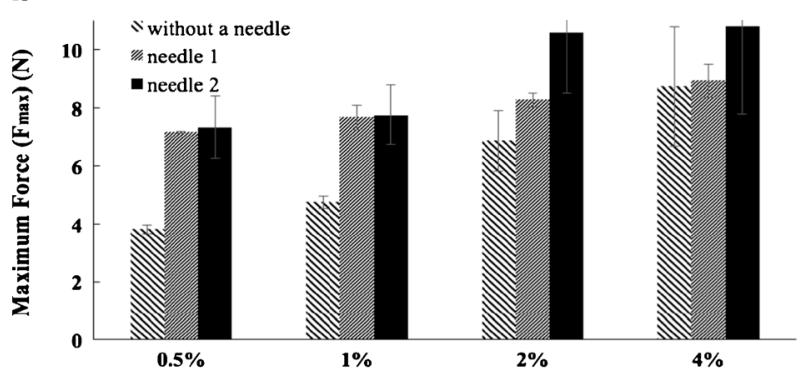

Concentration $(w / v)$

d

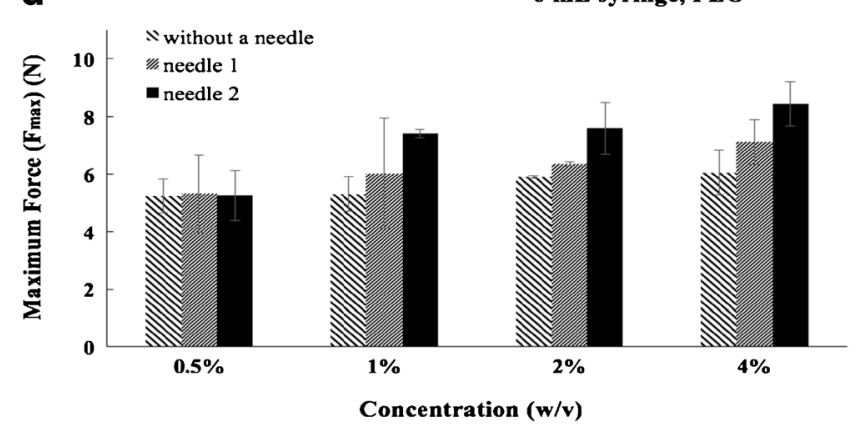

Fig. 8. Maximum force $\left(F_{\max }\right)$ values for different solution concentrations of HPMC and PEO using 3- and 6-mL syringes with and without needles $(N=3)$. The results are expressed as the mean of three experiments \pm standard deviation 
Table I. Total Work Done to Completely Extrude the HPMC Solutions Out of 3- and 6-mL Syringes with Attached Needle 1 (18G, 0.84 mm), Needle $2(21 \mathrm{G}, 0.51 \mathrm{~mm})$ and Without a Needle

\begin{tabular}{|c|c|c|c|c|c|c|c|c|}
\hline \multirow[b]{2}{*}{ Work (J) } & \multicolumn{2}{|l|}{$0.5 \%$ HPMC } & \multicolumn{2}{|l|}{$1 \%$ HPMC } & \multicolumn{2}{|l|}{$2 \% \mathrm{HPMC}$} & \multicolumn{2}{|l|}{ 4\% HPMC } \\
\hline & $3 \mathrm{~mL}$ & $6 \mathrm{~mL}$ & $3 \mathrm{~mL}$ & $6 \mathrm{~mL}$ & $3 \mathrm{~mL}$ & $6 \mathrm{~mL}$ & $3 \mathrm{~mL}$ & $6 \mathrm{~mL}$ \\
\hline Without a needle & $24.03 \pm 1.54$ & $128.35 \pm 15.6$ & $27.94 \pm 0.94$ & $148.33 \pm 14.4$ & $30.95 \pm 0.78$ & $161.52 \pm 4.32$ & $36.34 \pm 4.50$ & $185.6 \pm 3.08$ \\
\hline Needle 1 & $26.73 \pm 1.82$ & $148.89 \pm 4.61$ & $31.07 \pm 0.90$ & $170.61 \pm 7.49$ & $34.19 \pm 0.19$ & $173.23 \pm 1.68$ & $49.51 \pm 0.10$ & $199.14 \pm 0.24$ \\
\hline Needle 2 & $34.98 \pm 2.24$ & $170.15 \pm 5.01$ & $36.64 \pm 0.99$ & $202.53 \pm 7.72$ & $44.69 \pm 0.57$ & $231.10 \pm 4.46$ & $87.28 \pm 4.08$ & $350.49 \pm 13.30$ \\
\hline
\end{tabular}

Results are expressed as the mean \pm standard deviation

HPMC hydroxypropyl methylcellulose

\section{DISCUSSION}

In general, flow of viscous liquid through a cylindrical pipe is the relative motion of adjacent concentric layers of liquid within the pipe. Flow through a syringe as plunger force is applied follows the same principle. The shear stress produced by the plunger force is linear from zero along the central axis to a maximum value at the wall, while the shear rate varies nonlinearly and flow velocity is nearly zero at the wall and maximum along the central axis as shown in the Fig. 10 , and such behavior is dictated by the rheology of the formulation.

Overall, the rheological property of the pseudoplastic systems and their flow can be analyzed with the following exponential equation (29) (Eq. 5):

$F^{N}=\dot{\eta} G \ldots$

where " $F^{N}$ " is shearing stress required to produce a definite shearing rate " $G$ "; the term " $\eta$ " is a viscosity coefficient. The exponent " $N$ " rises as the flow tends toward a non-Newtonian and when $N=1$, the flow is Newtonian. Based on the volume of the syringe (i.e., 3 or $6 \mathrm{~mL}$ ), its barrel diameter and bore diameter and length of the needles used, biologics, and biopharmaceuticals may demonstrate variety of nonNewtonian behavior during syringeability/injectability and their functionality will be especially affected by the particle number, size and size distribution, shape, zeta potential and electroviscous effects, and viscosity behavior of the internal (disperse) phase of the dispersion system. Both HPMC and PEO solutions at different concentrations have a strong impact on the rheological characteristics of the process. Thus, viscous and complex biologics and their functionality including syringeability and injectability behavior require methodical examination.

\section{CONCLUSION}

With advances in formulation design, chemical and physical stabilization, and manufacturability, many biologicals and mAbs have become major biopharmaceutical products in the recent past for the treatment of cancer and many autoimmune diseases and have been successfully commercialized. Many of these highly concentrated formulations (>100 mg/mL, depending on the molecular structure and composition) are designed for subcutaneous delivery and present viscosity behavior that can change exponentially as concentration of the drug per milliliter increases. Highly concentrated systems present major concerns during syringeability and injectability especially as these products are typically stored refrigerated and temperature causes viscosity to increase significantly. Results of this study indicate that the use of parameter "total work done" " $W_{\mathrm{T}}$ " which entails the entire energy required to completely extrude the syringe content is a more precise assessment when compared to generally used factors like " $F_{\max }$ " or "DGF" in describing the flow behavior of pseudoplastic systems subjected to syringeability and injectability. It is shown that total work done " $W_{\mathrm{T}}$ " to inject viscous solutions of polymers studied will vary with solution concentration, bore diameter of the needle, and syringe size (both barrel diameter and volume). Changing the needle bore diameter alone (i.e., from larger to smaller) will disproportionately change the

Table II. Total Work Done to Completely Extrude PEO Solutions Out of 3- and 6-mL Syringes with Attached Needle 1 (18G, $0.84 \mathrm{~mm}$ ), Needle $2(21 \mathrm{G}, 0.51 \mathrm{~mm})$ and Without a Needle

\begin{tabular}{|c|c|c|c|c|c|c|c|c|}
\hline \multirow[b]{2}{*}{ Work (J) } & \multicolumn{2}{|l|}{$0.5 \%$ PEO } & \multicolumn{2}{|l|}{$1 \%$ PEO } & \multicolumn{2}{|l|}{$2 \% \mathrm{PEO}$} & \multicolumn{2}{|l|}{$4 \%$ PEO } \\
\hline & $3 \mathrm{~mL}$ & $6 \mathrm{~mL}$ & $3 \mathrm{~mL}$ & $6 \mathrm{~mL}$ & $3 \mathrm{~mL}$ & $6 \mathrm{~mL}$ & $3 \mathrm{~mL}$ & $6 \mathrm{~mL}$ \\
\hline Without a needle & $28.82 \pm 0.52$ & $107.95 \pm 12.85$ & $31.26 \pm 1.61$ & $115.86 \pm 1.61$ & $39.85 \pm 2.54$ & $130.72 \pm 0.56$ & $45.01 \pm 2.84$ & $140.97 \pm 11.14$ \\
\hline Needle 1 & $33.25 \pm 3.37$ & $126.68 \pm 5.81$ & $38.92 \pm 0.12$ & $135.42 \pm 1.23$ & $47.26 \pm 2.36$ & $138.76 \pm 2.00$ & $49.65 \pm 2.23$ & $169.22 \pm 5.74$ \\
\hline Needle 2 & $45.80 \pm 1.75$ & $145.51 \pm 2.98$ & $48.25 \pm 0.39$ & $175.84 \pm 3.89$ & $54.28 \pm 1.34$ & $214.62 \pm 1.34$ & $101.84 \pm 5.89$ & $376.91 \pm 4.78$ \\
\hline
\end{tabular}

Results are expressed as the mean \pm standard deviation $P E O$ polyethylene oxide 
a

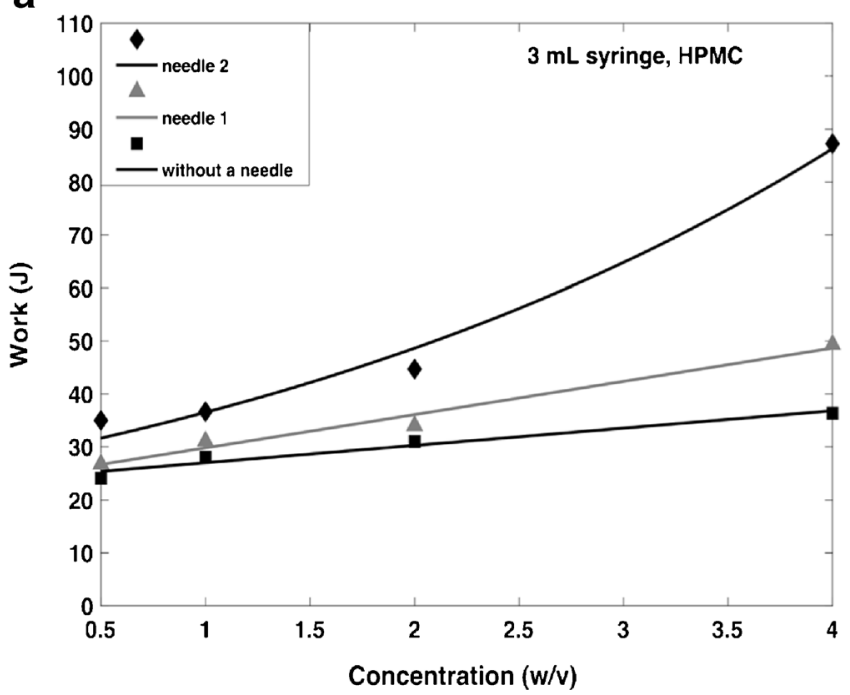

C

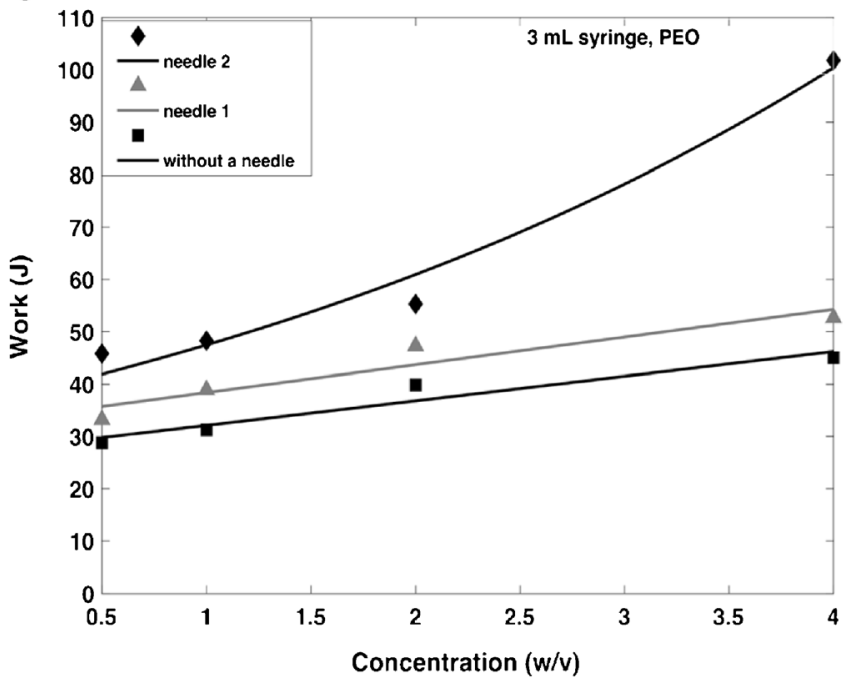

b

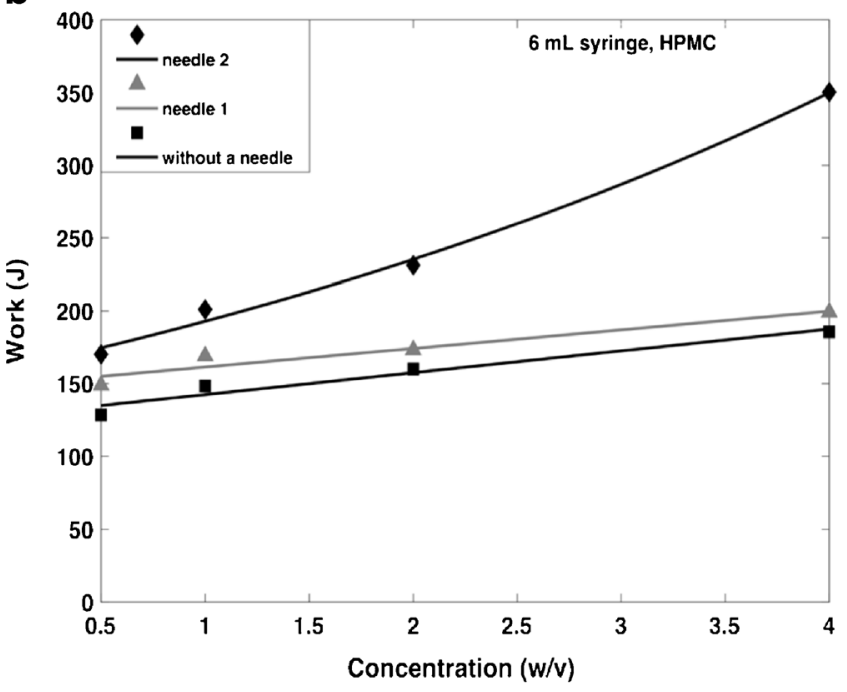

d

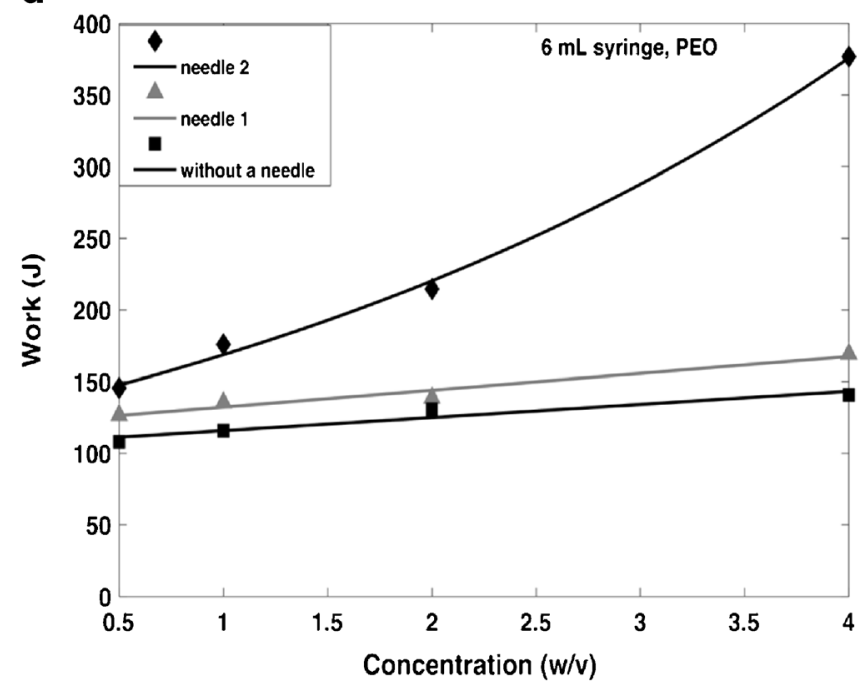

Fig. 9. Total work done to extrude different concentrations of HPMC and PEO from 3- and 6-mL syringes with and without the needles $(N=3)$

value of total work done to extrude the same material at the same flow rate. Both HPMC and PEO in general at the concentrations used appear to provide a convenient viscous system (exhibiting shear thinning behavior) representative of most injectable biopharmaceuticals in terms of their flow behavior. The $W_{\mathrm{T}}$ is significantly impacted by the needle inner diameter and syringe barrel volume/ dimensions as well as the injection rate (flow rate). This is further accentuated by the formulation composition, drug molecular structure and concentration $/ \mathrm{mL}$, product complexity, and its rheological properties with unpredictable magnitudes on syringeability and/or injectability and overall product functionality. It is suggested that the syringe-needle-biologic formulation in the context of syringeability and/or injectability presents numerous uncertainties and the device designers, pharmaceutical

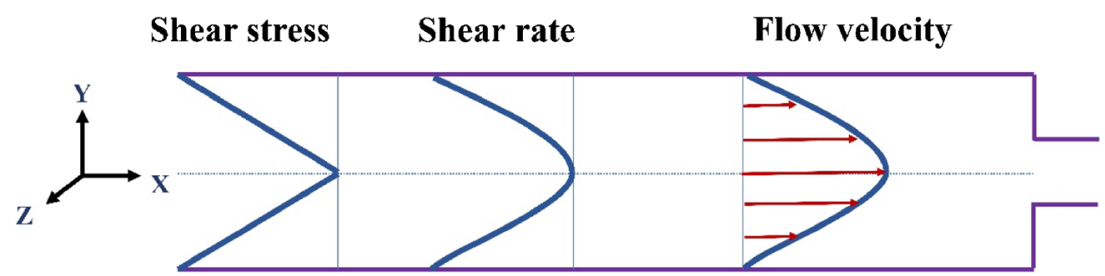

Fig. 10. Schematic representation of dynamics of flow through the syringe barrel showing linear (shear stress) and nonlinear (shear rate) relationship impacting flow velocity at the wall and central axis 
manufacturers, and syringe suppliers must fully examine functionality of the product in cohort before administration to the patients.

Open Access This article is distributed under the terms of the Creative Commons Attribution 4.0 International License (http://creativecommons.org/licenses/by/4.0/), which permits unrestricted use, distribution, and reproduction in any medium, provided you give appropriate credit to the original author(s) and the source, provide a link to the Creative Commons license, and indicate if changes were made.

\section{REFERENCES}

1. Mitragotri S, Burke PA, Langer R. Overcoming the challenges in administering biopharmaceuticals: formulation and delivery strategies. Nat Rev Drug Discov. 2014;13(9):655-72. https:// doi.org/10.1038/nrd4363.

2. Cleland JL, Powell MF, Shire SJ. The development of stable protein formulations: a close look at protein aggregation, deamidation, and oxidation. Crit Rev Ther Drug Carrier Syst. 1992;10(4):307-77.

3. Kanai S, Liu JU, Patapoff TW, Shire SJ. Reversible selfassociation of a concentrated monoclonal antibody solution mediated by Fab-Fab interaction that impacts solution viscosity. J Pharm Sci. 2008;97(10):4219-27. https://doi.org/10.1002/ jps.21322.

4. Rathore N, Pranay P, Bernacki J, Eu B, Ji W, Walls E. Characterization of protein rheology and delivery forces for combination products. J Pharm Sci. 2012;101(12):4472-80. https://doi.org/10.1002/jps.23297.

5. Rajagopal K, Wood J, Tran B, Patapoff TW, Nivaggioli T. Trehalose limits BSA aggregation in spray-dried formulations at high temperatures: implications in preparing polymer implants for long-term protein delivery. J Pharm Sci. 2013;102(8):265566. https://doi.org/10.1002/jps.23634.

6. Allison SD, Chang B, Randolph TW, Carpenter JF. Hydrogen bonding between sugar and protein is responsible for inhibition of dehydration-induced protein unfolding. Arch Biochem Biophys. 1999;365(2):289-98. https://doi.org/10.1006/ abbi.1999.1175.

7. Sasahara K, McPhie P, Minton AP. Effect of dextran on protein stability and conformation attributed to macromolecular crowding. J Mol Biol. 2003;326(4):1227-37. https://doi.org/ 10.1016/S0022-2836(02)01443-2.

8. Kerwin BA. Polysorbates 20 and 80 used in the formulation of protein biotherapeutics: structure and degradation pathways. J Pharm Sci. 2008;97(8):2924-35. https://doi.org/10.1002/jps.21190.

9. Ritschel WA, Suzuki K. In vitro testing of injectability. Pharm Ind. 1979;41:468-75.

10. Dexter MB, Shott MJ. The evaluation of the force to expel oily injection vehicles from syringes. J Pharm Pharmacol. 1979;31(1):497-500. https://doi.org/10.1111/j.20427158.1979.tb13570.x.

11. Monkos K. Concentration and temperature dependence of viscosity in lysozyme aqueous solutions. Biochim Biophys Acta (BBA)-Protein Struct Mol Enzymol. 1997;1339(2):304-10. https://doi.org/10.1016/S0167-4838(97)00013-7.
12. Monkos K. Viscosity analysis of the temperature dependence of the solution conformation of ovalbumin. Biophys Chem. 2000;85(1):7-16. https://doi.org/10.1016/S0301-4622(00)00127-7.

13. Tang Q, Munro PA, McCarthy OJ. Rheology of whey protein concentrate solutions as a function of concentration, temperature, pH and salt concentration. J Dairy Res. 1993;60(3):349-61. https://doi.org/10.1017/S0022029900027692.

14. Einstein A. Eine neue bestimmung der moleküldimensionen. Ann Phys. 1906;324(2):289-306. https://doi.org/10.1002/ andp.19063240204.

15. Krieger IM, Dougherty TJ. A mechanism for non-Newtonian flow in suspensions of rigid spheres. Trans Soc Rheol. 1959;3(1):137-52. https://doi.org/10.1122/1.548848.

16. Ravivarapu HB, Moyer KL, Dunn RL. Sustained suppression of pituitary-gonadal axis with an injectable, in situ forming implant of leuprolide acetate. J Pharm Sci. 2000;89(6):732-41. https:// doi.org/10.1002/(SICI)1520-6017(200006)89:6<732::AIDJPS4>3.0.CO;2-D.

17. Chen M, Gupta V, Anselmo AC, Muraski JA, Mitragotri S. Topical delivery of hyaluronic acid into skin using SPACEpeptide carriers. J Control Release. 2014;173:67-74. https:// doi.org/10.1016/j.jconrel.2013.10.007.

18. Muthu MS, Rawat MK, Mishra A, Singh S. PLGA nanoparticle formulations of risperidone: preparation and neuropharmacological evaluation. Nanomedicine. 2009;5(3):323-33. https:// doi.org/10.1016/j.nano.2008.12.003.

19. Brodbeck KJ, Pushpala S, McHugh AJ. Sustained release of human growth hormone from PLGA solution depots. Pharm Res. 1999;16(12):1825-9. https://doi.org/10.1023/ A:1018943107688.

20. Food and Drug Administration. Guidance for industry, container closure systems for packaging human drugs and biologics. United States Department of Health and Human Services. 1999:1-56.

21. ICH S. Test procedures and acceptance criteria for new drug substances and new drug products: chemical substances. International Conference on Harmonisation, IFPMA, Geneva;1999.

22. Overcashier DE, Chan EK, Hsu CC. Technical considerations in the development of prefilled syringes for protein products. Am Pharm Rev. 2006;9(7):77-83.

23. Fry A. Injecting highly viscous drugs. Pharm Technol. 2014;38:8-10.

24. Chemistry of METHOCEL ${ }^{\mathrm{TM}}$ cellulose ethers-a technical review. ht t p:// m sdssearch.dow.com/

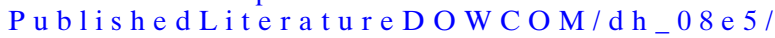
0901b803808e5f58.pdf? filepath=dowwolff/pdfs/noreg/19802289.pdf\&fromPage=GetDoc. Accessed 23 Oct 2017.

25. POLYOX TM water soluble resins combining flexibility with consistency. http://msdssearch.dow.com/

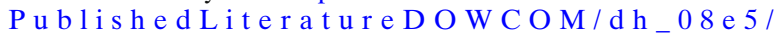
0901b803808e5467.pdf? filepath=dowwolff/pdfs/noreg/32600108.pdf\&fromPage=GetDoc. Accessed 23 Oct 2017.

26. Cilurzo F, Selmin F, Minghetti P, Adami M, Bertoni E, Lauria S, et al. Injectability evaluation: an open issue. AAPS PharmSciTech. 2011;12(2):604-9. https://doi.org/10.1208/s12249011-9625-y.

27. Wang $\mathrm{N}, \mathrm{Wu}$ J. Maximizing the user's benefit in the mobile cloud computing. In: Proceedings of the Eighth Wireless of the Students, by the Students, and for the Students Workshop 2016 Oct 3. ACM, pp. 21-23.

28. Wang N, Wu J. Opportunistic wifi offloading in a vehicular environment: waiting or downloading now? In Computer communications, IEEE INFOCOM 2016-The 35th Annual IEEE International Conference on 2016 Apr 10. IEEE, pp. 1-9.

29. Alfred M, James D, Camarata A. Physical chemical principles in the pharmaceutical sciences. Physical Pharmacy Lea \& Febiger Washington Square. 1983;600. 